\title{
Adatom Kinetics On and Below the Surface: The Existence of a New Diffusion Channel
}

\author{
Jörg Neugebauer, Tosja K. Zywietz, and Matthias Scheffler \\ Fritz-Haber-Institut der Max-Planck-Gesellschaft, Faradayweg 4-6, D-14195 Berlin, Germany \\ John E. Northrup \\ Palo Alto Research Center, 3333 Coyote Hill Road, Palo Alto, California 94304 \\ Huajie Chen and R. M. Feenstra \\ Department of Physics, Carnegie Mellon University, Pittsburgh, Pennsylvania 15213
}

(Received 5 June 2002; published 6 February 2003)

\begin{abstract}
Employing density-functional theory in combination with scanning tunneling microscopy, we demonstrate that a thin metallic film on a semiconductor surface may open an efficient and hitherto not expected diffusion channel for lateral adatom transport: adatoms may prefer diffusion within this metallic layer rather than on top of the surface. Based on this concept, we interpret recent experiments: We explain why and when In acts as a surfactant on GaN surfaces, why Ga acts as an autosurfactant, and how this mechanism can be used to optimize group-III nitride growth.
\end{abstract}

Most known transport mechanisms on semiconductor surfaces have rather high activation temperatures: typically a ratio between activation and melting temperature well above 0.5 is found $\left(T_{\text {act }} / T_{\text {melt }}>0.5\right)$. Growing at lower temperature typically results in a rough surface morphology. Recently, a number of interesting transport mechanisms having low activation energies have been discussed. One example is the diffusion of transition metal atoms into bulk $\mathrm{Si}$, which may actually be more efficient than diffusion on the surface [1]. Surface morphology changes mediated by bulk vacancy transport [2] and vacancy mediated surface diffusion [3] have also been discussed recently for metals. In this Letter, we discuss a growth mechanism in which the surface transport involves adatom diffusion below a metallic adlayer on a semiconductor surface. Employing state-of-the art first principles calculations and scanning tunneling microscopy, we demonstrate that adatoms may prefer to incorporate and diffuse between the adlayer and the substrate. This mechanism, which we call adlayer enhanced lateral diffusion (AELD) becomes activated already at rather low temperatures, thereby enabling the growth of materials having a high melting temperature even at modest temperatures.

The materials system for which this mechanism is demonstrated are the group-III nitrides $(\mathrm{GaN}, \mathrm{InN}$, AlN, and their alloys). The members of this technologically important class of materials are strongly bonded and exhibit high melting temperatures. For example [4], $\mathrm{GaN}$ has a melting temperature of $2791 \mathrm{~K}$ and so the optimum growth temperature is expected to be higher than $1400 \mathrm{~K}$. However, since $\mathrm{GaN}$ decomposes in vacuum when the temperature exceeds $1200 \mathrm{~K}$, low pressure growth methods such as molecular beam epitaxy (MBE) can be performed only at temperatures well below the decomposition temperature. One might therefore ex- pect that MBE growth results in a poor surface morphology and indeed, this is found for a large range of possible growth conditions. Only under extreme Ga-rich conditions, close to the onset of Ga-droplet formation, can smooth surfaces be achieved [5,6]. Recent experimental studies indicate that the presence of an In adlayer also leads to a smooth surface morphology and a better crystal quality [7-10].

In order to understand why the presence of In or excess Ga leads to smoother surfaces, it is essential to know how these atoms modify the surface reconstruction and how the modified surface affects adatom kinetics. To address these issues, we performed total-energy calculations and geometry optimizations employing density-functional theory within the local-density approximation (LDA). Test calculations using a generalized gradient approximation [11] gave essentially the same results as the LDA with energy differences $\leq 0.1 \mathrm{eV}$. For details of the method, see Ref. [12]. Soft Troullier-Martins pseudopotentials [13] are employed for $\mathrm{Ga}, \mathrm{N}$, and In. The $\mathrm{Ga} 3 d$ and In $4 d$ electrons were explicitly treated as valence electrons. The surfaces were modeled by a repeated slab geometry (slab thickness: four $\mathrm{GaN}$ double layers) where one side of the slab was passivated by fractional pseudohydrogen. The atoms in the first two double layers of the slab and the adatoms were relaxed. Relaxations below the two double layers have been found to be negligible. A $(3 \times 3)$ Monkhorst-Pack mesh was used to sample the Brillouin zone [14] together with a plane-wave energy cutoff of $70 \mathrm{Ry}$.

Let us start by considering how In modifies the structure of $\mathrm{GaN}$ surfaces. In thermodynamic equilibrium this requires the identification of the surface having the lowest energy. The surface formation energy is given by

$$
E^{\text {surf }}=E_{\text {slab }}^{\text {tot }}-n_{\mathrm{Ga}} \mu_{\mathrm{Ga}}-n_{\mathrm{In}} \mu_{\mathrm{In}}-n_{\mathrm{N}} \mu_{\mathrm{N}},
$$


where $E_{\text {slab }}^{\text {tot }}$ is the total energy of a thin slab of the material, $n_{\alpha}$ is the number of atoms of species $\alpha$ in the slab, and $\mu_{\alpha}$ is the corresponding chemical potential. In thermodynamic equilibrium the chemical potentials of the three species are constrained to vary within a certain range of values. Under conditions in which In behaves as a surfactant, i.e., no In is incorporated in the growing film, the film consists entirely of $\mathrm{GaN}$ and we have the constraint $\mu_{\mathrm{Ga}}+\mu_{\mathrm{N}}=\mu_{\mathrm{GaN}}$, where $\mu_{\mathrm{GaN}}$ is the chemical potential of bulk GaN. It follows that only one potential $\left(\mu_{\mathrm{Ga}}\right.$ or $\left.\mu_{\mathrm{N}}\right)$ can be chosen freely. Here we choose the Ga chemical potential. We obtained the upper limits on the N, $\mathrm{Ga}$, and In chemical potentials by calculating the energy per atom for the $\mathrm{N}_{2}$ molecule and for the orthorhombic (Ga) and body-centered-tetragonal (In) phases of $\mathrm{Ga}$ and In. Combining this formalism with total-energy calculations for a large number of possible structures (with varying numbers of $\mathrm{Ga}$ and In atoms in the first and second surface layers), a phase diagram of all relevant equilibrium structures (Fig. 1) has been constructed.

Under In poor conditions the phase diagram reproduces the previously identified reconstructions of the clean $\mathrm{GaN}$ (0001) surface: For extreme Ga-rich conditions the laterally contracted Ga-bilayer structure [15] is stable, and for less Ga-rich conditions the $\mathrm{Ga}$ and $\mathrm{N}$ adatom structures $[16,17]$ are stable. For high In concentrations (corresponding to a higher In chemical potential), most of the bare GaN surfaces become unstable and structures having an In adlayer (Fig. 1) appear. Under extreme N-rich conditions In is present in both the first and second layers [8]. Based on the calculated phase diagram, it becomes possible to relate surface structures to experimental growth conditions. In particular, we are able to identify the surfaces leading to optimum $\mathrm{GaN}$ growth. As has been discussed above, optimum growth is observed under extreme Ga-rich conditions [i.e., in a region of the phase diagram where the Ga-bilayer structure is stable (Fig. 1)] or in the presence of In, i.e., in a region where the In-adlayer structure is stable. Therefore, we identify two main structures leading to smooth growth: the In adlayer and the Ga bilayer. Both structures contain two or more

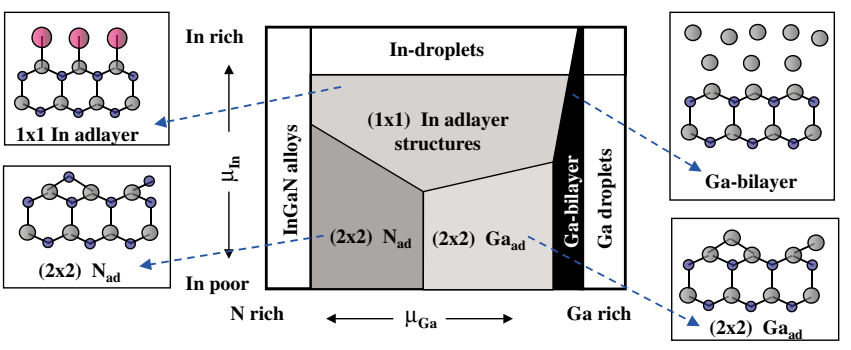

FIG. 1 (color online). Phase diagram of possible equilibrium surface reconstructions of bare and In-covered GaN (0001) as a function of the In and $\mathrm{Ga}$ chemical potentials. Also shown is the schematic geometry (side view) of the relevant surface structures. Small, medium, and large sized filled circles mark the positions of $\mathrm{N}, \mathrm{Ga}$, and In atoms, respectively. layers of metal atoms. It is therefore tempting to conclude that the formation of a thin metal film leads to a more efficient diffusion channel.

In order to test this hypothesis, we have calculated diffusion pathways and migration energy barriers for $\mathrm{Ga}$ and $\mathrm{N}$ adatoms on the In-adlayer surface and compare these with prior calculations for bare $\mathrm{GaN}$ (0001) (i.e., in the absence of the In adlayer) [18]. For Ga adatoms on top of the In adlayer, we find qualitatively the same adsorption sites and diffusion pathway as on bare GaN (0001). The main difference is the magnitude of the diffusion barrier: It is $0.12 \mathrm{eV}$ (on the In-adlayer structure) compared to $0.7 \mathrm{eV}$ [on bare $\mathrm{GaN}$ (0001)]. Thus, the presence of the In adlayer significantly enhances the mobility of $\mathrm{Ga}$ adatoms.

For $\mathrm{N}$ adatoms we find a qualitatively different behavior. Calculating the adsorption energy of $\mathrm{N}$ for a large number of possible configurations shows that $\mathrm{N}$ prefers to sit below the top surface layer, i.e., between the Ga and In layers (Fig. 2, left). As can be seen in the contour plot, the $\mathrm{N}$ adatom forms strong bonds to the underlying Ga layer. The N-Ga bond length is slightly stretched (by 7\% compared to the bulk bond length) due to the metallic environment. In contrast, the bonds of the $\mathrm{N}$ atom to the upper layer are rather weak: The bond length is more than $30 \%$ larger than in InN bulk.

Based on the identified local minima for the $\mathrm{N}$ adatom, we have systematically explored its migration paths and diffusion barriers. We thereto calculated the potentialenergy surface $E_{\mathrm{tot}}^{\mathrm{PES}}\left(\vec{R}_{\|}\right)$, where the lateral position $\vec{R}_{\|}$ of the adatom is kept fixed while its height and the coordinates of atoms in the first three surface layers were allowed to relax. Using this approach two potentialenergy surfaces for adatom diffusion emerge: For the first the adatom resides mainly on top of the In adlayer. For the second the adatom is always below the adlayer. The first case corresponds to "normal" on-surface adsorption which has been typically regarded as the main mechanism for lateral transport at the surface. The second one corresponds to a new mechanism (we call it

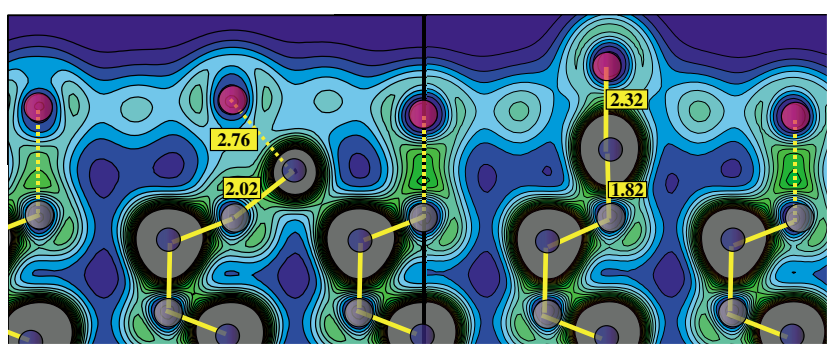

FIG. 2 (color online). Cross-sectional view of the valence charge density for a nitrogen adatom at the stable subsurface site (left) and at the barrier configuration for subsurface diffusion (right). Grey and dark grey (online color: blue) filled circles mark the position of $\mathrm{Ga}$ and $\mathrm{N}$ atoms, respectively. The balls in the top layer mark In atoms. The numbers give the bond length in $\AA$. 
adlayer enhanced lateral diffusion) which has so far not been considered in previous work.

The potential-energy surface (PES) for the adlayer enhanced lateral diffusion is shown in Fig. 3. The calculations have been performed using a $3 \times 3$ surface unit cell. As can be seen, the energetically most stable site is a hollow site. The barrier for diffusion along a path passing directly underneath an In atom is $0.5 \mathrm{eV}$. We note that at high temperatures concerted interaction may modify this value. In contrast, diffusion of $\mathrm{N}$ adatoms on the bare surface occurs with a barrier of $1.3 \mathrm{eV}$ [18]. An analysis of the PES for on-surface diffusion showed that diffusion barriers are significantly larger $(\geq 1.5 \mathrm{eV})$ and that this PES is connected to the PES for AELD: For both PES the energetically most stable adatom configuration is the subsurface $(\mathrm{H} 3)$ hollow site. From the latter result it is obvious that $\mathrm{N}$ adatoms diffuse without any barrier from on surface to subsurface. This, together with the fact that the diffusion barrier for AELD is lowest, implies that adlayer enhanced lateral diffusion is the dominant and most efficient diffusion channel for $N$ adatoms on the Interminated GaN (0001) surface. Moreover, since the barrier for $\mathrm{N}$ adatom diffusion on the bare surface is much higher, $1.3 \mathrm{eV}$ [18], it is clear that the In adlayer significantly reduces the diffusion barrier and enables smooth growth of $\mathrm{GaN}$ to occur at much lower temperatures.

Insight into the low energy of the AELD barrier can be obtained by analyzing the charge density shown in Fig. 2. In the stable configuration (Fig. 2, left) the N-In interaction is weak. However, in the barrier configuration (Fig. 2, right) the N-In distance is reduced by $16 \%$ and a strong N-In bond is formed. In going from the stable to the barrier configuration the $\mathrm{N}$ adatom has to break two out of three Ga-N bonds which, in the absence of the In

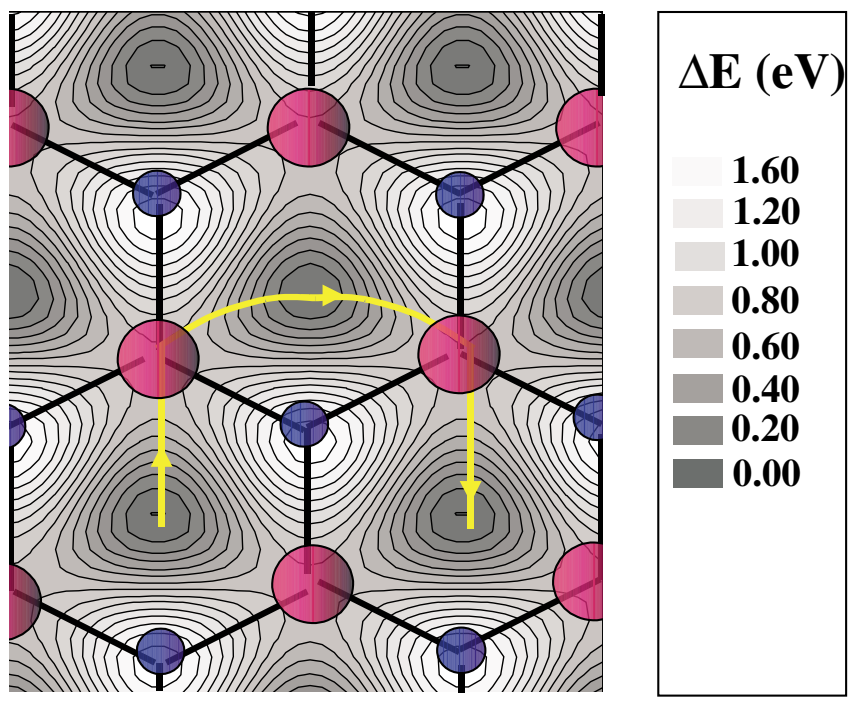

FIG. 3 (color online). Potential energy surface for a subsurface $\mathrm{N}$ adatom at the In adlayer GaN (0001) surface. The line with arrows marks the lowest energy diffusion path. The large/ small filled circles indicate $\mathrm{In} / \mathrm{N}$ atoms. adlayer, costs roughly $3 \mathrm{eV}$ [17]. The creation of the strong In- $\mathrm{N}$ bond in the barrier configuration largely compensates for these broken bonds and explains the strong reduction in the diffusion barrier.

In order to demonstrate the presence of the AELD effect experimentally, we have studied growth on Interminated (0001) and (0001) surfaces of GaN. A comparison of results from these surfaces is ideal for our purpose since their topmost layers have identical geometry (i.e., for on-surface diffusion similar diffusion barriers are expected) whereas the second layer consists of $\mathrm{Ga}$ or N, respectively. According to our previous discussion only for the first case, where two metallic layers are present, is AELD expected to be operational. To realize these surfaces an In-adlayer structure has been grown using plasma-assisted molecular beam epitaxy on $\mathrm{GaN}(0001)$ and $(000 \overline{1})$. In both cases, the surfaces have been grown under $\mathrm{N}$-rich conditions (i.e., the growth surfaces are rough for both polarities, in the absence of In) and with incident In flux greater than incident $\mathrm{Ga}$ flux. Such conditions are needed in order to obtain Incovered surfaces. A detailed description of experimental details and growth conditions can be found elsewhere $[8,19,20]$. STM images of the two surfaces are shown in Figs. 4(a) and 4(b). Auger electron spectroscopy measurements for both surfaces indicate close to one monolayer of In terminating the surfaces $[8,20]$. Through a combination of these experimental results together with a theoretical analysis of the type discussed above, we can confidently assign the surface structures as pictured in Figs. 4(c) and 4(d). The In on GaN(0001) forms an adlayer, with sparse occupation of In in the underlying Ga layer. In contrast, on the (0001) surface, the In atoms substitute for $\mathrm{Ga}$ atoms in the top terminating layer, forming the structure pictured in Fig. 4(d) in which the In atoms are bonded to $\mathrm{N}$ atoms below, and again there is a sparse occupation of In in the underlying Ga layer. In both Figs. 4(a) and 4(b) the brighter corrugation maxima arise from top layer In atoms which have In atoms positioned in an underlying layer, thereby pushing up the top In atoms by $\approx 0.05 \AA[19,20]$.

Having realized two surfaces with the desired properties (i.e., with a single and a double metallic layer), we can now examine and interpret the difference in their kinetic behavior as evidenced in their surface morphology. Figures 4(e) and 4(f) show atomic force microscopy (AFM) images of the morphology of $\approx 100 \mathrm{~nm}$ thick In-terminated $\mathrm{GaN}$ films, both grown at temperatures of about $650{ }^{\circ} \mathrm{C}$. As described previously, GaN (0001) films grown under N-rich conditions display rough morphology $[5,8]$. However, as seen in Fig. 4(e), the presence of a surface consisting of two metallic layers (which can be realized by the addition of even a small amount of In) causes the morphology to become smooth. In contrast, for the $\mathrm{GaN}(000 \overline{1})$ films, where the surface consists only of a single metallic layer, the rough morphology which occurs during growth under $\mathrm{N}$-rich conditions is found to persist. 

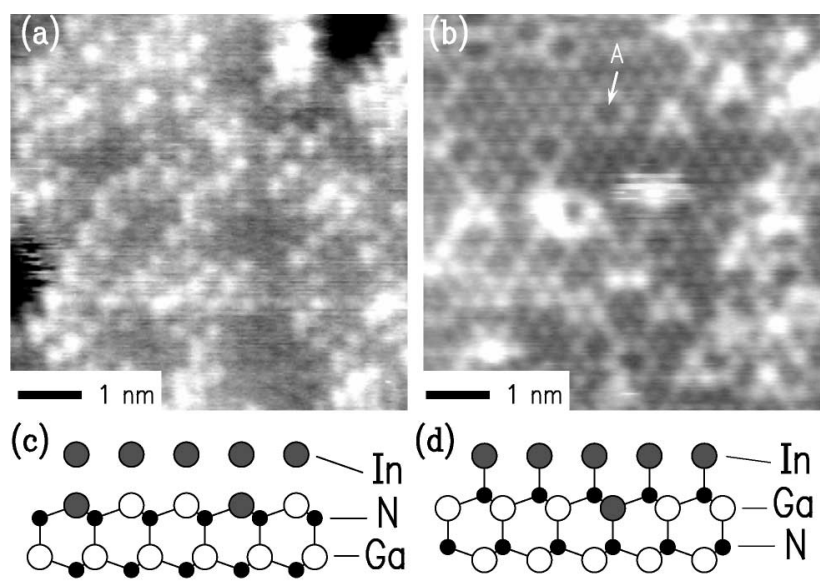

(d)
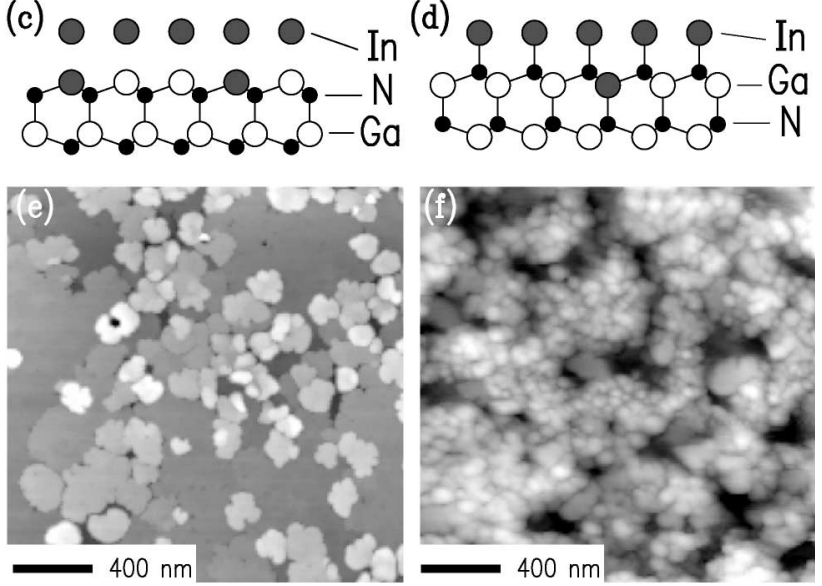

FIG. 4. Experimental results for In-terminated GaN films, comparing (0001) (left side) and (0001) (right side) surfaces. (a),(b) STM images, for films terminated with a layer of In atoms. The feature "A" in (b) arises from a Ga atom in the top layer, with all other top layer atoms being In. The bright corrugation maxima in both images arise from In atoms in underlying layers. The images were acquired with sample voltages of +1.25 and $-1.0 \mathrm{~V}$, respectively, and are displayed with gray scale ranges of 0.5 and $0.8 \mathrm{~nm}$, respectively. (c),(d) Side view models for the surface structures. (e),(f) AFM images of the surface morphology of InGaN films. The images are displayed with gray scale ranges of 15 and $23 \mathrm{~nm}$, respectively.

The surface diffusivity is thus found to be significantly higher on the $\mathrm{GaN}$ (0001) compared to the (0001) surface, and we tentatively attribute this difference to the AELD mechanism. Other surface kinetic mechanisms could also differ between the (0001) and (0001) surfaces, although a recent work [21] indicating that desorption from $\mathrm{GaN}(0001)$ surfaces is relatively independent of the specific surface structure does support our interpretation (i.e., it implies that our differing morphologies are not simply a result of a surface-induced change in the desorption rate).

An interesting issue is the generality of the AELD mechanism. Factors which are important for it to be operative are (i) the presence of a thin metallic film on the surface (consisting of at least two atomic layers) and (ii) adatoms which prefer subsurface rather than onsurface adsorption sites. These conditions have (so far) not been observed on the widely studied $\mathrm{P}$, As, or Sbbased III-V compound semiconductors, although those 056101-4 studies have been primarily performed on (001)-oriented surfaces which are stabilized by dimers, and future studies on (111) surfaces may reveal the AELD mechanism. Another technologically important class of materials where AELD might be operative are oxides, e.g., for $\mathrm{Zn}$-rich $\mathrm{ZnO}$ or Al-rich $\mathrm{Al}_{2} \mathrm{O}_{3}$ growth. Also, the mechanism could occur on metal surfaces containing a surfacesegregated layer of some other species. The preference for subsurface vs on-surface adsorption sites is expected to be satisfied when the atomic radius of the adsorbates is significantly smaller than the radius of the host adatoms. In addition to crystal growth, the AELD mechanism may also be operative in situations involving the distribution of adsorbates on a surface, e.g., enabling the efficient transport of species to a step edge where catalytic reactions may occur.

We thank B. Daudin (CEA France), C. Kruse, D. Hommel (Uni Bremen), and H. Riechert (Infineon) for many stimulating discussions. We also acknowledge financial support from the "Deutsche Forschungsgemeinschaft: SFB296" (J. N.). The work at CMU was supported by grants from the National Science Foundation and the Office of Naval Research. Work at the Palo Alto Research Center was supported by the Air Force Office of Scientific Research.

[1] M. Y. Lee and P. A. Bennett, Phys. Rev. Lett. 75, 4460 (1995).

[2] F. K. McCarty, J. A. Nobel, and N.C. Bartelt, Nature (London) 412, 622 (2001).

[3] R. van Gastel et al., Nature (London) 408, 665 (2000).

[4] J. Karpinski, J. Jun, and S. J. Porowski, J. Cryst. Growth 66, 1 (1984).

[5] E. J. Tarsa et al., J. Appl. Phys. 82, 5472 (1997).

[6] R. Averbeck and H. Riechert, Phys. Status Solidi A 176, 301 (1999).

[7] F. Widmann et al., Appl. Phys. Lett. 73, 2642 (1998).

[8] H. Chen et al., J. Vac. Sci. Technol. B 18, 2284 (2000).

[9] C. Adelmann et al., Appl. Phys. Lett. 75, 3518 (1999).

[10] C. Kruse et al., Appl. Phys. Lett. 79, 3425 (2001).

[11] J. P. Perdew, K. Burke, and M. Ernzerhof, Phys. Rev. Lett. 77, 3865 (1996).

[12] M. Bockstedte et al., Comput. Phys. Commun. 107, 187222 (1997).

[13] N. Troullier and J. L. Martins, Phys. Rev. B 43, 1993 (1991).

[14] H. J. Monkhorst and J. D. Pack, Phys. Rev. B 13, 5188 (1976).

[15] J. E. Northrup et al., Phys. Rev. B 61, 9932 (2000).

[16] A. R. Smith et al., Phys. Rev. Lett. 79, 3934 (1997).

[17] A. R. Smith et al., Surf. Sci. 423, 70 (1999).

[18] T. K. Zywietz, J. Neugebauer, and M. Scheffler, Appl. Phys. Lett. 73, 487 (1998).

[19] H. Chen et al., Phys. Rev. Lett. 85, 1902 (2000).

[20] H. Chen et al., MRS Internet J. Nitride Semicond. Res. 6, 11 (2001).

[21] C. Adelmann et al., J. Appl. Phys. 91, 9638 (2002) 